\title{
A NONLINEAR ERGODIC THEOREM FOR A REVERSIBLE SEMIGROUP OF NONEXPANSIVE MAPPINGS IN A HILBERT SPACE
}

\author{
WATARU TAKAHASHI
}

\begin{abstract}
Let $C$ be a nonempty closed convex subset of a Hilbert space, $S$ a right reversible semitopological semigroup, $S=\left\{T_{t}: t \in S\right\}$ a continuous representation of $S$ as nonexpansive mappings on a closed convex subset $C$ into $C$, and $F(S)$ the set of common fixed points of mappings $T_{t}, t \in S$. Then we deal with the existence of a nonexpansive retraction $P$ of $C$ onto $F(S)$ such that $P T_{t}=T_{t} P=P$ for each $t \in S$ and $P x$ is contained in the closure of the convex hull of $\left\{T_{t} x: t \in S\right\}$ for each $x \in C$.
\end{abstract}

1. Introduction. Let $H$ be a real Hilbert space with norm $\|\cdot\|$ and inner product $\langle\cdot, \cdot\rangle$ and $C$ a nonempty closed convex subset of $H$. A mapping $T: C \rightarrow C$ is called nonexpansive [3] on $C$, if

$$
\|T x-T y\| \leq\|x-y\| \quad \text { for every } x, y \in C .
$$

We denote by $F(T)$ the set of fixed points of $T$. The first nonlinear ergodic theorem for nonexpansive mappings was established in 1975 by Baillon [1]: Let $C$ be a closed convex subset of a Hilbert space and $T$ a nonexpansive mapping of $C$ into itself. If the set $F(T)$ is nonempty, then for each $x \in C$, the Cesàro means

$$
S_{n}(x)=\frac{1}{n} \sum_{k=0}^{n-1} T^{k} x
$$

converge weakly to some $y \in F(T)$. In this case, putting $y=P x$ for each $x \in$ $C, P$ is a nonexpansive retraction of $C$ onto $F(T)$ such that $P T=T P=P$ and $P x \in \overline{\operatorname{co}}\left\{T^{n} x: n=0,1,2, \ldots\right\}$ for each $x \in C$, where $\overline{\text { co }} A$ is the closure of the convex hull of $A$. In $[\mathbf{1 1}]$, the author proved the existence of such a retraction"ergodic retraction"-for an amenable semigroup of nonexpansive mappings in a Hilbert space. Then, Hirano and Takahashi [5] extended this result to a Banach space. On the other hand, Rodé [10] found a sequence of means on the semigroups, generalizing the Cesàro means on positive integers, such that the corresponding sequence of mappings converges to a retraction onto the set of common fixed points. Recently Lau [7] considered the problem of weak convergence for a right reversible semigroup of nonexpansive mappings.

In this paper, we deal with the existence of "ergodic retraction" for a right reversible semigroup of nonexpansive mappings, that is, we prove a nonlinear ergodic theorem for such a semigroup in a Hilbert space. This theorem is a generalization

Received by the editors December 4, 1984 and, in revised form, May 1, 1985.

1980 Mathematics Subject Classification. Primary 47A35, 47H09.

Key words and phrases. Ergodic theorem, reversible semigroup, nonexpansive mapping, fixed point. 
of the author's result [11]. In the proof, we also give a characterization of "ergodic retraction".

2. Nonlinear ergodic theorem. Let $S$ be a semitopological semigroup, i.e., $S$ is a semigroup with a Hausdorff topology such that for each $a \in S$ the mappings $s \rightarrow a \cdot s$ and $s \rightarrow s \cdot a$ from $S$ to $S$ are continuous. $S$ is called right reversible if any two closed left ideals of $S$ have nonvoid intersection. In this case, $(S, \leq)$ is a directed system when the binary relation " $\leq$ " on $S$ is defined by $a \leq b$ if and only if $\{a\} \cup \overline{S a} \supseteq\{b\} \cup \overline{S b}, a, b \in S$.

Right reversible semitopological semigroups include all commutative semigroups and all semitopological semigroups which are right amenable as discrete semigroups; see $[4,6$, p. 335]. Let $C$ be a closed convex subset of a real Hilbert space $H$ and $S=\left\{T_{s}: s \in S\right\}$ a continuous representation of $S$ as nonexpansive mappings on a closed convex set $C$ into $C$, i.e., $T_{a b}(x)=T_{a} T_{b}(x), a, b, \in S, x \in C$ and the mapping $(s, x) \rightarrow T_{s}(x)$ from $S \times C$ into $C$ is continuous when $S \times C$ has the product topology. Let $F(S)$ denote the set $\left\{x \in C: T_{s} x=x\right.$ for all $\left.s \in S\right\}$ of common fixed points of mappings $T_{s}, s \in S$ in $C$. Then, as is well known, $F(S)$ is a closed convex subset of $C$. Let $Q$ be the metric projection of $H$ onto $F(S)$. Then, by Phelps [9], $Q$ is nonexpansive. Now we prove a nonlinear ergodic theorem for a right reversible semigroup of nonexpansive mappings in a Hilbert space.

THEOREM. Let $C$ be a nonempty closed convex subset of a real Hilbert space $H$, $S$ a right reversible semitopological semigroup and $S=\left\{T_{s}: s \in S\right\}$ a continuous representation of $S$ as nonexpansive mappings of a closed convex set $C$ into $C$. Suppose that

$$
F(S)=\bigcap\left\{F\left(T_{s}\right): s \in S\right\} \neq \varnothing .
$$

Then the following are equivalent:

(a) $\bigcap_{s \in S} \overline{\operatorname{co}}\left\{T_{t} x: t \geq s\right\} \cap F(S) \neq \varnothing$ for each $x \in C$.

(b) There is a nonexpansive retraction $P$ of $C$ onto $F(S)$ such that $P T_{t}=T_{t} P=$ $P$ for every $t \in S$ and $P x \in \overline{\operatorname{co}}\left\{T_{t} x: t \in S\right\}$ for every $x \in C$.

Proof. (b) $\Rightarrow(\mathrm{a})$. Let $x \in C$. Then $P x \in F(S)$. Also

$$
P x \in \bigcap_{s \in S} \overline{\operatorname{co}}\left\{T_{t} x: t \geq s\right\} \text {. }
$$

In fact,

$$
P x=P T_{s} x \in \overline{\operatorname{co}}\left\{T_{t} T_{s} x: t \in S\right\} \subset \overline{\operatorname{co}}\left\{T_{t} x: t \geq s\right\}
$$

for every $s \in S$.

(a) $\Rightarrow(\mathrm{b})$. Let $x \in C$ and $f \in F(S)$. Let $b \geq a$. Then, since $b \in\{a\} \cup \overline{S a}$, we may assume $b \in \overline{S a}$. Let $\left\{s_{\alpha}\right\}$ be a net in $S$ such that $s_{\alpha} a \rightarrow b$. Then, for each $\alpha$,

$$
\left\|T_{s_{\alpha} a} x-f\right\|^{2}=\left\|T_{s_{\alpha}}\left(T_{a} x\right)-T_{s_{\alpha}} f\right\|^{2} \leq\left\|T_{a} x-f\right\|^{2} .
$$

Hence, $\left\|T_{b} x-f\right\|^{2} \leq\left\|T_{a} x-f\right\|^{2}$. So the $\lim _{s}\left\|T_{s} x-f\right\|^{2}$ exists. Let

$$
g(f)=\lim _{s}\left\|T_{s} x-f\right\|^{2} \quad \text { for every } f \in F(S)
$$

and

$$
r=\inf \{g(f): f \in F(S)\}
$$


Then, since the real-valued function $g$ on $F(S)$ is convex and continuous and $g(f) \rightarrow$ $\infty$ as $\|f\| \rightarrow \infty$, from [2, p. 79], there exists $f_{0} \in F(S)$ with $g\left(f_{0}\right)=r$. Hence the set

$$
M(x)=\{f \in F(S): g(f)=r\}
$$

is nonempty. We now show that $M(x)$ consists of one point. In fact, let $f_{0}, f_{1} \in$ $M(x)$. Then using the parallelogram law, we obtain

$$
\left\|\frac{f_{0}-f_{1}}{2}\right\|^{2}=\frac{\left\|T_{s}-f_{0}\right\|^{2}}{2}+\frac{\left\|T_{s}-f_{1}\right\|^{2}}{2}-\left\|T_{s} x-\frac{f_{0}+f_{1}}{2}\right\|^{2}
$$

for every $s \in S$. So we have

$$
\left\|\frac{f_{0}-f_{1}}{2}\right\|^{2}=r-\lim _{s}\left\|T_{s} x-\frac{f_{0}+f_{1}}{2}\right\|^{2} \leq 0
$$

and hence $f_{0}=f_{1}$.

Let $M(x)=\{u\}$ and let $Q$ be the metric projection of $H$ onto $F(S)$. Then from [7] we know that $Q T_{s} x$ converges strongly to some $z \in F(S)$. We show $u=z$. Let $a \leq b$. Then we may assume $b \in \overline{S a}$. Let $\left\{s_{\alpha}\right\}$ be a net in $S$ such that $s_{\alpha} a \rightarrow b$. Then for each $\alpha$,

$$
\left\|Q T_{a} x-T_{s_{\alpha} a} x\right\|^{2}=\left\|T_{s_{\alpha}} Q T_{a} x-T_{s_{\alpha}} T_{a} x\right\|^{2} \leq\left\|Q T_{a} x-T_{a} x\right\|^{2} .
$$

So, we have $\left\|Q T_{a} x-T_{b} x\right\|^{2} \leq\left\|Q T_{a} x-T_{a} x\right\|^{2}$. Hence if $a \leq b$, then

$$
\left\|Q T_{a} x-T_{b} x\right\|^{2} \leq\left\|Q T_{a} x-T_{a} x\right\|^{2} \leq\left\|f-T_{a} x\right\|^{2}
$$

for every $f \in F(S)$. Therefore, for any $a \in S$, we have

$$
g\left(Q T_{a} x\right)=\lim _{b}\left\|T_{b} x-Q T_{a} x\right\|^{2} \leq\left\|T_{a} x-f\right\|^{2}
$$

for every $f \in F(S)$ by above. Since $g$ is continuous and $Q T_{a} x$ converges strongly to $z \in F(S)$, we have $g(z) \leq \lim _{a}\left\|T_{a} x-f\right\|^{2}=g(f)$ for every $f \in F(S)$. Then $u=z=\lim _{t} Q T_{t} x$.

From (a), let $v \in F(S) \cap \bigcap_{s \in S} \overline{\operatorname{co}}\left\{T_{t} x: t \geq s\right\}$. Then, since

$$
\|u-v\|^{2}=\left\|T_{s} x-v\right\|^{2}-\left\|T_{s} x-u\right\|^{2}-2\left\langle u-v, T_{s} x-u\right\rangle
$$

for every $s \in S$, we have

$$
\|u-v\|^{2}+2 \lim _{s}\left\langle u-v, T_{s} x-u\right\rangle=\lim _{s}\left\|T_{s} x-v\right\|^{2}-\lim _{s}\left\|T_{s} x-u\right\|^{2} \geq 0 .
$$

Let $\varepsilon>0$. Then we have

$$
2 \lim _{s}\left\langle u-v, T_{s} x-u\right\rangle>-\|u-v\|^{2}-\varepsilon .
$$

Hence there exists $s_{0} \in S$ such that

$$
2\left\langle u-v, T_{s} x-u\right\rangle>-\|u-v\|^{2}-\varepsilon
$$

for every $s \geq s_{0}$. Since $v \in \overline{c o}\left\{T_{t} x: t \geq s_{0}\right\}$, we have

$$
2\langle u-v, v-u\rangle \geq-\|u-v\|^{2}-\varepsilon
$$

This inequality implies $\|u-v\|^{2} \leq \varepsilon$. Since $\varepsilon$ is arbitrary, we have $u=v$. Therefore

$$
F(S) \cap \bigcap_{s \in S} \overline{\operatorname{co}}\left\{T_{t} x: t \geq s\right\}=\{u\}
$$


Set $P x=\lim _{t} Q T_{t} x$ for every $x \in C$. Then we have $T_{s} P x=P x$ and

$$
P T_{s} x=\lim _{t} Q T_{t} T_{s} x=\lim _{t} Q T_{t s} x=P x
$$

for every $s \in S$ and $x \in C$. From $\{P x\}=F(S) \cap \bigcap_{s \in S} \overline{\operatorname{co}}\left\{T_{t} x: t \geq s\right\}$, it is obvious that $P x \in \overline{c o}\left\{T_{s} x: s \in S\right\}$ for each $x \in C$. Since

$$
\|P x-P y\|=\lim _{t}\left\|Q T_{t} x-Q T_{t} y\right\| \leq\|x-y\|
$$

for every $x, y \in C$, it follows that $P$ is nonexpansive.

Lau and Takahashi $[8]$ obtained an analogous result in a Banach space. However the Theorem is sharper than $[8]$. The following corollary was actually proved in [11].

COROLLARY. Let $C$ be a nonempty closed convex subset of a real Hilbert space $H$ and $S$ an amenable semigroup of nonexpansive mappings t of $C$ into itself. Suppose that

$$
F(S)=\bigcap\{F(t): t \in S\} \neq \varnothing \text {. }
$$

Then, there exists a nonexpansive retraction $P$ of $C$ onto $F(S)$ such that $P t=$ $t P=P$ for every $t \in S$ and $P x \in \overline{\mathrm{co}}\{t x: t \in S\}$ for every $x \in C$.

Proof. Let $\mu$ be an invariant mean on $S$ and $x \in C$. Then, since $F(S) \neq \varnothing$, $\{t x: t \in S\}$ is bounded and hence for each $y$ in $H$, the real-valued function $t \mapsto$ $\langle t x, y\rangle$ is bounded. Denote by $\mu_{t}\langle t x, y\rangle$ the value of $\mu$ at the function. Then this is linear and continuous in $y$. So by the Riesz theorem, there exists an $x_{0} \in H$ such that $\mu_{t}\langle t x, y\rangle=\left\langle x_{0}, y\right\rangle$ for every $y \in H$ and the point $x_{0}$ is contained in $F(S) \cap \bigcap_{s \in S} \overline{c 0}\{t x: t \geq s\}$. For details, see $[\mathbf{1 1}]$.

\section{REFERENCES}

1. J. B. Baillon, Un théorème de type ergodique pour les contractions non linéaires dans un espace de Hilbert, C. R. Acad. Sci. Paris Sér. A-B 280 (1975), 1511-1514.

2. V. Barbu and Th. Precupanu, Convexity and optimization in Banach spaces, Editura Academiei R.S.T., Bucharest, 1978.

3. F. E. Browder, Nonexpansive nonlinear operators in a Banach space, Proc. Nat. Acad. Sci. U.S.A. 54 (1965), 1041-1044.

4. M. M. Day, Amenable semigroups, Illinois J. Math. 1 (1957), 509-544.

5. N. Hirano and W. Takahashi, Nonlinear ergodic theorems for an amenable semigroup of nonexpansive mappings in a Banach space, Pacific J. Math. 112 (1984), 333-346.

6. R. D. Holmes and A. T. Lau, Nonexpansive actions of topological semigroups and fixed points, J. London Math. Soc. (2) 5 (1972), 330-336.

7. A. T. Lau, Semigroup of nonexpansive mappings on a Hilbert space, J. Math. Anal. Appl. 105 (1985), 514-522.

8. A. T. Lau and W. Takahashi, Weak convergence and nonlinear ergodic theorems for reversible semigroups of nonexpansive mappings (to appear).

9. R. R. Phelps, Convex sets and nearest points, Proc. Amer. Math. Soc. 8 (1957), 790-797.

10. G. Rodé, An ergodic theorem for semigroups of nonexpansive mappings in a Hilbert space, J. Math. Anal. Appl. 85 (1982), 172-178.

11. W. Takahashi, A nonlinear ergodic theorem for an amenable semigroup of nonexpansive mappings in a Hilbert space, Proc. Amer. Math. Soc. 81 (1981), 253-256.

Department of Information SCiEnCEs, Tokyo Institute of TeChNology, OHOKAYAMA, MEgURO-KU, TOKYO, JAPAN 